\title{
Evaluación de la Formación Profesional para el Empleo en la Ciudad Autónoma de Melilla (España)
}

\author{
Joaquín Arana Torres*
} $M^{a}$ Carmen Olmos Gómez ***

\section{RESUMEN:}

Elestudio que a continuación se ofrece resume las investigaciones realizadas dentro del estudio realizado en la Formación Profesional Ocupacional del Servicio Público de Empleo Estatal, del aprendizaje continuo y ocupacional, englobando no sólo los aspectos formativos sino su vinculación con respecto a la inserción, estableciendo una evaluación formativa para la posterior adecuación de la vinculación entre la Formación Profesional del SPEE y la Formación Profesional del MEC en la Ciudad Autónoma de Melilla para la elaboración de propuestas que fomente el empleo y la formación y por tanto el desarrollo formativo-laboral de la ciudad.

\section{Palabras Clave:}

Empleo, inserción laboral, formación ocupacional y desarrollo formativo-laboral

\section{AbSTRACT:}

This paper is a summary of a research about the Occupational Formation in the Estate Public Job Service, Continuous learning and occupational aspects encom-

* Departamento Económico, Financiero y Contabilidad. U. de Granada • quinoarana@hotmail.com

** Departamento Métodos de Investigación y Diagnóstico en Educación. U. de Granada • molmos@ugr.es 
passing not only training but its link with respect to integration, establishing a formative assessment for the subsequent adjustment of the linkage between the SPEE and Vocational Training of MEC in the City of Melilla to the development of proposals to promote employment and training and therefore training and labor development of the city.

\section{KEYWORDS:}

Employment, job placement, occupational training, and labor development.

\section{MARCO TEÓRICO:}

\section{1.- LA FORMACIÓN PROFESIONALIZADORA}

La reestructuración económica en diferentes sectores de actividad, cambio de las cualificaciones, el impulso arrollador de las nuevas tecnologías de la información, el fenómeno de la mundialización económica...; todos estos factores conforman nuevos retos para las políticas de empleo y de formación, donde se demandan distintas estrategias frente al desempleo.

Al mismo tiempo, la transición entre escuela-trabajo también ha variado y está definida por tres elementos interconectados (según el Grup d'Estudis Sociologics sobre la vida quotidiana i el treball, 2000):

a) El acceso al trabajo es lento y dificultoso y no es suficiente con la titulación académica, lo que hace que los itinerarios formativos sean largos y complejos.

b) Nuevas formas de organización del trabajo alteran la antigua transición profesional.

c) La nueva estructuración del mercado de trabajo acentúa el diferencia entre la oferta y la demanda.

Llegados a este punto, no resta más que adentrarse en el problema del cambio y de las necesidades que se generan y que influyen en las características que adquiere la formación. Dicho de otra manera, debemos reflexionar acerca de los desafíos a los cuales se enfrentan los sistemas de formación para responder a las situaciones cambiantes y a las necesidades planteadas por la sociedad.

Concretaremos los desafíos que se le presentan a la formación en cinco grandes retos, partiendo de la idea de que están muy relacionados entre sí y que el primer reto es el que sirve de estructuración en torno al cual se imbrican los demás: 
1.- La formación ante el empleo, reto fundamental donde se pretende comprobar la relación existente entre el binomio formación y empleo, estudiando las dos posturas existentes en torno a este tema, que son por un lado la visión de la formación como parte estratégica de la política de empleo, correlacionada directamente con la empleabilidad de las personas y por otro la visión de la formación como condición necesaria, pero no suficiente, para llevar a cabo una buena política de empleo. A partir de estas dos posturas intentaremos explicar cual es nuestra postura frente al tema.

2.- La formación ante el reto del cambio tecnológico, cuyo objetivo es conocer como afectan los cambios tecnológicos a la formación y, al mismo tiempo, estudiar la respuesta que puede dar la formación para abordar dichos cambios.

3.- La formación ante la cualificación profesional, donde estudiaremos el papel que juega la formación respecto a la cualificación profesional de las personas, partiendo del análisis de la definición del término cualificación y de su influencia en el desarrollo de los recursos humanos.

4.- La formación ante los nuevos yacimientos de empleo, en este reto se trata de revisar los nuevos factores que justifican y avalan los nuevos yacimientos de empleo, para conocer cual es la respuesta que da la formación ante este hecho.

5.- La formación ante la organización del trabajo, el propósito del cual es estudiar el papel que la formación ha de tomar frente a las nuevas formas de gestión, dirección y organización del sistema productivo que invade a las empresas.

Por ello, en la primera vía de intermediación y de acceso al empleo como son las redes sociales de parentesco, amistad y vecindad, es más importante la densidad y la calidad de las relaciones que la formación en sí. En la segunda forma de acceso, la intermediación institucional pública (Servicio Publico de Empleo Estatal, SPEE), la formación juega un papel más importante, ya que constituye un criterio de selección y competencia entre los candidatos al empleo, sin que ello signifique realmente el uso de su cualificación. En la tercera forma, la intermediación privada (a través de las empresas...), el papel de la formación no suele jugar un papel determinante, ya que el acceso al empleo puede estar mediatizado por otras variables como la confianza inspirada en la entrevista o las necesidades urgentes de fuerza de trabajo.

Podríamos concluir que, el mérito de la formación no es suficiente, pero sí actúa como mecanismo de preselección, de modo que, en algunos casos, la 
formación es una condición necesaria pero no suficiente para acceder a determinados empleos, pues en ocasiones tienen más que ver con características individuales (actitudinales y comportamentales) de quienes buscan empleo, alejadas de su formación.

Podemos interpretar entonces que la formación sirve de criba, sin embargo el mayor nivel educativo no supone mayor trabajo cualificado, sino que las empresas contratan trabajadores con niveles educativos superiores a la cualificación exigida en el trabajo a desempeñar

Nuestra idea respecto al tema es que la formación se convierte en la fórmula contra la exclusión del mercado laboral de muchos trabajadores, pues existe una probada relación entre los niveles de formación adquiridos y su empleabilidad, esto supone, al mismo tiempo, una relación entre educación, desarrollo económico y empleo, apoyada por algunos autores. Realmente los análisis estadísticos realizados por la OCDE (2002) muestran que:

- A menor nivel de formación mayor tasa de desempleo, tanto en hombres como en mujeres.

- A mayor nivel de formación mayor nivel de participación en la población activa y menor tasa de desempleo tanto en hombres como en mujeres.

\section{ENSEÑANZA POR COMPETENCIAS EN FORMACIÓN. COMPETENCIAS GE-} NÉRICAS Y TRANSVERSALES

\subsection{CONCEPTO DE COMPETENCIA}

Una competencia es "una característica subyacente en una persona que está causalmente relacionada con el desempeño, referido a un criterio superior en un trabajo o situación" (Spencer y Spencer, 1993).

De acuerdo con esta definición, hablamos de característica subyacente porque la competencia es una parte profundamente arraigada en la personalidad del estudiante que puede predecir su comportamiento en una amplia variedad de situaciones académicas o profesionales; destacamos que está causalmente relacionada porque puede explicar o predecir su futuro desempeño profesional; mientras que referido a un criterio significa que la competencia predice la actuación buena o deficiente del estudiante utilizando un estándar de medida específico (MEC, 2007).

Siguiendo con la definición del Ministerio de Educación y Ciencia las características subyacentes a la competencia son de diferentes tipos. Así, podemos hablar de motivos, rasgos de la personalidad, autoconcepto, conocimientos y habilidades: 
Los motivos son las cosas que un estudiante piensa de modo consistente o quiere como causa de determinada acción. Cuando un estudiante acude a clase, realiza una práctica en el laboratorio o prepara un examen lo hace para lograr metas tales como aprobar una materia, dominar determinadas habilidades o satisfacer una necesidad personal (p.e. reconocimiento, amistad, pertenencia a un grupo, etc.). De estos motivos el estudiante puede ser consciente en mayor o menor grado.

Los rasgos de la personalidad son características que se manifiestan físicamente y que suponen respuestas consistentes a situaciones o informaciones.

El autoconcepto refleja las actitudes, los valores o la propia imagen del estudiante. Hay estudiantes que se consideran líderes mientras que otros prefieren pasar desapercibidos; hay estudiantes que anteponen una calificación a cualquier cosa, en tanto que otros valoran de un modo preferente el compañerismo o el reconocimiento del grupo; hay estudiantes que se muestra una actitud apática ante determinadas materias o situaciones mientras que otros tienen una actitud de clara colaboración.

El conocimiento es la información con que cuenta una persona sobre áreas más o menos específicas de contenido de un plan de estudios. Esos conocimientos pueden estar referidos a conceptos, hechos o procedimientos ligados a las materias que estudian.

Finalmente, la habilidad es la destreza o capacidad del estudiante para desarrollar una cierta actividad física o mental. Así, un estudiante de medicina puede examinar el ojo sin producir daños al paciente o el estudiante de ingeniería puede diseñar las operaciones de una planta industrial que afectan a una decena de procesos y subprocesos diferentes.

Por tanto se define a la competencia, como el aprendizaje activo de conocimientos, habilidades, actitudes y capacidades que, relacionados entre sí, facultan a la persona para asumir los cambios y los nuevos roles en los distintos contextos socio-organizativos y satisfacer demandas complejas para el desempeño eficaz de una profesión, tarea, actividad o función.

El desarrollo de competencias en estudiantes y trabajadores ha tratado de abordarse desde varios enfoques que, en esencia, diferencian entre lo genérico (motivos, rasgos) y lo específico de las materias de estudio o de la profesión (conocimientos, habilidades). Así, las publicaciones de Alex (1991), Bunk (1994), Mertens (1996), o Echeverría (2005) vienen a incidir en esa diferenciación con mayor o menor claridad.

\section{2.- TIPOS DE MÉTODOS DIDÁCTICOS}

El método se concreta en una variedad de modos, formas, procedimientos, estrategias, técnicas, actividades y tareas de enseñanza y aprendizaje. En función de esta 
variedad se establecen diversos tipos de métodos: De ahí que hayan sido clasificados por los diferentes autores de formas distintas según el criterio o el aspecto de los mismos que consideraran de mayor importancia. Nosotros los hemos agrupado en tres bloques según el enfoque utilizado:

a. El Enfoque didáctico para la individualización. Este enfoque centra su atención en el estudiante en cuanto sujeto individual.

b. El enfoque de la socialización didáctica. Se centra en la dimensión social del proceso didáctico y los modelos de enseñanza.

c. El enfoque globalizado. Aglutina los métodos que pueden abordar interdisciplinarmente la realidad.

Por tanto, enseñar competencias implica saber intervenir en situaciones reales, que por ser reales, siempre son complejas (Zabala y Arnau, 2007).

\section{3.- Sistemas y PROCEDimientos de EVAluación}

Los "sistemas de evaluación" constituyen el último elemento de planificación y ejecución del proceso de enseñanza-aprendizaje según el modelo propuesto. Este modelo supone un cambio de paradigma (Tagg, 2003) al trasladar el centro de atención desde la enseñanza del profesor al aprendizaje del alumno. Como efecto directo de este cambio los sistemas de evaluación cobran especial protagonismo pues son el elemento principal que orienta y motiva el aprendizaje del alumno y la propia enseñanza.

\section{Principales Estrategias Evaluativas}

- Evaluación global al final de periodos amplios de formación (por ejemplo, después de los dos primeros cursos).

- Evaluación global al final de la titulación (memoria, proyecto, etc... con equivalencia en créditos, etc.).

- Evaluación acumulativa por materias sin evaluación global final ni por periodos amplios.

- Evaluación acumulativa por materias con evaluación global final y/o por periodos amplios. 


\section{Procedimientos y técnicas evaluativas}

- Pruebas objetivas (verdadero/falso, elección múltiple, emparejamiento de elementos,...).

- Pruebas de respuesta corta.

- Pruebas de respuesta larga, de desarrollo.

- Pruebas orales (individual, en grupo, presentación de temas-trabajos,...).

- Trabajos y proyectos.

- Informes/memorias de prácticas.

- Pruebas de ejecución de tareas reales y/o simuladas.

- Sistemas de Autoevaluación (oral, escrita, individual, en grupo).

- Escalas de actitudes (para recoger opiniones, valores, habilidades sociales y directivas, conductas de interacción, ...).

- Técnicas de observación (registros, listas de control, ...).

- Portafolio.

\section{3: EL MERCADO DE TRABAJO 2008}

Es importante estudiar quienes son los destinatarios finales de la Formación Profesional para el Empleo en la Ciudad de Melilla, por un lado los trabajadores desempleados ( Formación Ocupacional ) y trabajadores en activo ( Formación continua ) así como su relación con la estructura económica de la ciudad. Para ello presentamos algunos datos referidos a la estructura del desempleo y la estructura económica de la Ciudad Autónoma de Melilla.

Según la EPA, la tasa de actividad y la tasa de empleo disminuyó durante 2008 en ambos sexos, sin embargo, la tendencia de la tasa de paro ha sido diferente en cuanto a sexo, ha disminuido la tasa de paro de las mujeres y aumentado la tasa de paro de los hombres, lo que demuestra que las mujeres de Melilla se han incorporado en mayor medida al mercado laboral.

En comparación con los datos nacionales, hay que resaltar que en Melilla la tasa de paro ha disminuido en 8,69 puntos, mientras que en general en España ha aumentado en 5,31 puntos porcentuales.

La tendencia de la tasa de actividad es al alza a nivel nacional, sin embargo a nivel de la provincia de Melilla ha disminuido en 6,76 puntos. 


\begin{tabular}{|c|c|c|c|c|c|c|}
\hline \multicolumn{7}{|c|}{ TASAS DE ACTIVIDAD, EMPLEOY PARO } \\
\hline \multicolumn{7}{|c|}{ IVTRIMESTRE 2007} \\
\hline \multirow{2}{*}{ TASA } & \multicolumn{3}{|c|}{ MELILLA } & \multicolumn{3}{|c|}{ ESPAÑA } \\
\hline & Hombres & Mujeres & Total & Hombres & Mujeres & Total \\
\hline Actividad & 70,95 & 41,32 & 55,78 & 69,23 & 49,37 & 59,12 \\
\hline Empleo & 63,56 & 29,27 & 46,01 & 64,51 & 43,94 & 54,03 \\
\hline Paro & 10,41 & 29,15 & 17,52 & 6,83 & 11,00 & 8,60 \\
\hline \multicolumn{7}{|c|}{ IVTRIMESTRE 2008} \\
\hline \multirow{2}{*}{ TASA } & \multicolumn{3}{|c|}{ MELILLA } & \multicolumn{3}{|c|}{ ESPAÑA } \\
\hline & Hombres & Mujeres & Total & Hombres & Mujeres & Total \\
\hline Actividad & 66,03 & 33,64 & 49,02 & 69,21 & 51,38 & 60,13 \\
\hline Empleo & 56,22 & 26,81 & 40,76 & 60,24 & 43,60 & 51,77 \\
\hline Paro & 14,85 & 20,46 & 16,87 & 12,96 & 15,14 & $|3,9|$ \\
\hline
\end{tabular}

Fuente Instituto Nacional de Estadística (INE). Datos en miles de personas

La distribución de la población activa por sectores económicos muestra la gran importancia que tiene el sector servicios en nuestra Ciudad., algo más del $80 \%$ de la población activa se encuadra en él, con una diferencia de 16,10 puntos por encima de los datos nacionales.

Los sectores de la industria y la construcción se encuentran muy por debajo de las cifras nacionales y la agricultura no tiene prácticamente actividad en Melilla.

Hay que destacar el alto porcentaje de personas que se encuentran paradas buscando su primer empleo o han dejando el último hace más de un año (10\%) en comparación con el dato nacional (4,50\%)

\begin{tabular}{|c|c|c|c|c|}
\hline \multicolumn{5}{|c|}{ ACTIVOS POR SECTOR ECONÓMICO } \\
\hline \multirow{2}{*}{ SECTOR } & \multicolumn{3}{|c|}{ MELILLA } & \multicolumn{2}{c|}{ ESPAÑA } \\
\cline { 2 - 5 } & IV Trim. 2007 & IV Trim. 2008 & IV Trim. 2007 & IV Trim. 2008 \\
\hline \multirow{2}{*}{ Agricultura } & - & - & $4,60 \%$ & $4,1 \%$ \\
\hline Industria & $2,2 \%$ & $2,9 \%$ & $15,20 \%$ & $14,4 \%$ \\
\hline Construcción & $6,4 \%$ & $6,1 \%$ & $13,00 \%$ & $12,0 \%$ \\
\hline Servicios & $80,8 \%$ & $81,0 \%$ & $64,00 \%$ & $64,9 \%$ \\
\hline Parados(*) & $10,6 \%$ & $10,0 \%$ & $3,10 \%$ & $4,5 \%$ \\
\hline
\end{tabular}

(*) Parados que buscan su primer empleo o han dejado el último hace más de un año. Fuente Instituto Nacional de Estadística (INE). Datos en miles de personas 
Durante el año 2008 el número total de trabajadores se ha visto incrementado en un 21,30\%, sin embargo, el número de empresas ha descendido ligeramente, un 0,34\%.

El incremento en el número de trabajadores ha sido general en todos los sectores, pero el más beneficiado ha sido el sector de servicios, el cual ha incrementado esta cifra en mayor proporción.

En el mes de Diciembre de 2008, según datos de la Tesorería General de la Seguridad Social, existían un 23,04\% más de afiliados a la seguridad social en este sector. Las empresas, sin embargo, se han visto incrementadas en menor medida, y en el sector servicios a 31 de Diciembre de 2.008 aparecían de alta nueve empresas menos que en el mismo mes del año anterior.

Después de analizar todos los datos anteriores, vemos que la estructura empresarial en Melilla continúa teniendo como base fundamental el sector servicios, englobando un 88,30\% del número de trabajadores y un 82,24\% del número de empresas.

El sector primario y la industria tienen muy poco peso y apenas tienen actividad en Melilla.

EMPRESAS

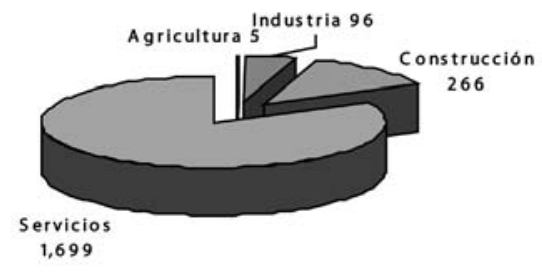

TRABAJADORES

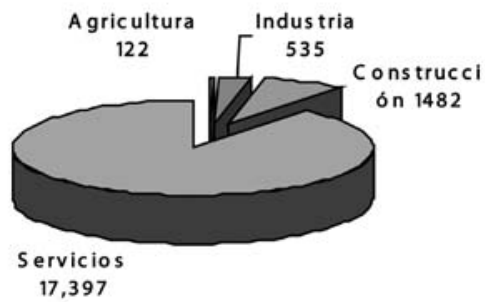

Fuente: MTIN. Cuentas de cotización y Trabajadores afiliados a la Seguridad Social. Diciembre 2008

\section{LA INVESTIGACIÓN}

\section{1.- PRESENTACiÓN:}

META:

El presente estudio pretende mejorar la inserción laboral de los usuarios de formación ocupacional en la Ciudad Autónoma de Melilla.

Para ello haremos: 


\subsection{1.- Objetivos}

Evaluar la inserción laboral de los desempleados/as, que en los últimos dos años han participado en cursos de formación ocupacional.

Adecuar los diseños de formación ocupacional en Melilla al nuevo Subsistema de Formación Profesional para el Empleo de 2007.

Las acciones específicas para el segundo objetivo se concretan a su vez en:

Acotar el diseño y desarrollo de cursos de formación ocupacional a los estudios de Tendencias de Mercado de Trabajo de Melilla.

. Ponderar y priorizar la formación destinada al fomento de nuevos yacimientos de empleo (población mayor, personas discapacitadas, infancia, inmigrantes y colectivos con riesgo de exclusión social.

Generar y apoyar formación por competencias para nuevos empresarios mediante acciones de promoción, apoyo y asesoramiento del autoempleo.

\subsection{2.- Metodología:}

\section{A) Para el OBJETIVO 1:}

Evaluar la calidad de la formación que han recibido los desempleados/as que en los últimos dos años han participado en cursos de formación ocupacional.

DISEÑO:

a) EVALUACIÓN DE NECESIDADES que conllevará la descripción de los participantes e integración de sus aportaciones, (recabaremos información de empresarios, empleados, formadores para integrar sus manifestaciones en orden a construir una priorización de sus necesidades utilizando métodos de clasificación ordenada a partir de criterios de importancia, urgencia y consecuencias.

b) REQUERIMIENTOS METODOLOGICOS DEL PLAN ( elaboración de encuestas y cuestionarios que comprendan los constructos teóricos implícitos en la meta y objetivos de la investigación. Obtendremos evidencias de su validez teórica, lógica, y empírica.

c) ANALISIS DE DATOS. Una vez observadas las evidencias de fiabilidad y validez con que podemos hacer adecuadas interpretaciones e inferencias con los datos que obtengamos, procederemos a organizar la información para su análisis utilizando los programas y estadísticos que mejor explican los objetivos que perseguíamos.

Ordenados los resultados de los análisis se procede a la exposición de resultados bien interpretados. Antes de concluir expresaremos las limitaciones que se han tenido en el desarrollo de la investigación 


\subsection{3.- Muestra:}

Selección de la muestra. Características.

La selección de la muestra se realizó, por un procedimiento de selección aleatoria sistemática.

En la tabla siguiente se muestra las empresas resultantes para el presente estudio, el cual se ha organizado en seis sectores.

CUADRO No 36- ESTRUCTURA DE LA MUESTRA.

\section{POBLACIÓN PARTICIPANTE:}

- El número de alumnos a los que se procedió a realizar la encuesta fue de 91 personas desempleadas.

Contestaron a la encuestación los participantes de 11 cursos, que a continuación se describen, repartidos en 8 centros colaboradores.

\section{2.- ANÁLISIS DE RESUlTAdos}

ANALISIS DE DATOS. Una vez observadas las evidencias de fiabilidad y validez con que podemos hacer adecuadas interpretaciones e inferencias con los datos obtenidos se procede a organizar la información para su análisis utilizando los programas y estadísticos que mejor explican los objetivos que perseguíamos.

Ordenados los resultados de los análisis se procede a la exposición de resultados bien interpretados.

Cuadro Explicativo Del Diseño De La Investigación

\begin{tabular}{|l|l|}
\hline OBJETIVOS & TÉCNICA DE RECOGIDA \\
\hline $\begin{array}{l}\text { OBJETIVO 1: Evaluar la inserción laboral de los } \\
\text { desempleados/as, que en los últimos dos años han } \\
\text { participado en cursos de formación ocupacional. }\end{array}$ & $\begin{array}{l}\text { Análisis documental } \\
\text { Cuestionarios }\end{array}$ \\
\hline $\begin{array}{l}\text { OBJETIVO 2: Adecuar los diseños de formación } \\
\text { Ocupacional en Melilla a la nueva Normativa 2008. }\end{array}$ & $\begin{array}{l}\text { Análisis documental } \\
\text { Cuestionarios } \\
\text { Entrevistas }\end{array}$ \\
\hline
\end{tabular}




\section{5.- CONCLUSIONES}

Vinculados a los objetivos de la investigación:

\section{OBJETIVO No 1 .}

Evaluar la calidad de la formación que han recibido los desempleados/as, que en los últimos dos años han participado en cursos de formación ocupacional.

Hay que resaltar que este trabajo de investigación ofrece, a tenor de los resultados estadísticos, que en términos generales el grado de satisfacción que experimentan los desempleados al termino de la realización de un curso de formación es alto, pues la moda, o el grado de la variable estudiada que mayor frecuencia absoluta poseía en cada uno de los ítems correspondientes a la encuesta, rara vez bajaba de 3, es decir, lo normal eran puntuaciones en cada una de las preguntas de la encuesta situadas en un intervalo de 3 a 5 .

Las distribuciones de frecuencias obtenidas al pasar las encuestas al programa SPSS, arrojan en términos generales porcentajes validos siempre superiores al valor 3 , considerado en todos los ítems de la encuesta como el valor medio de las diferentes opciones presentadas

Este trabajo de investigación que muestra la configuración del mercado de trabajo en Melilla y arroja índices de calidad de los cursos de formación ocupacional que realiza el Servicio Publico de Empleo Estatal ( SPEE), proporciona información concreta y muy útil sobre el desarrollo de los cursos y sobre todo de la gestión del centro colaborador del SPEE. Existe una pregunta que muestra las posibilidades de inserción a juicio de las personas que realizan los cursos, en concreto se refiere a las expectativas que tiene en el desempleado la realización del curso para la obtención de empleo. Este dato seria muy útil ponerlo en relación con la inserción profesional al término de cada curso de formación, pues nos ofrecería a posteriori si existe correlación estadística entre la formación ocupacional y el empleo. La correlación entre FORMACION Y EMPLEO constituye un fin en si mismo por parte de las autoridades gubernativas y mas en un mercado de trabajo como el de la Ciudad de Melilla. Por tanto este trabajo debe continuarse con una parte posterior que es mostrar si existe relación entre los cursos y el empleo, a través de los datos de la inserción profesional.

\section{OBJETIVO No 2}

Adecuar los diseños de formación ocupacional en Melilla al nuevo Subsistema de Formación Profesional para el Empleo de 2007.

Hay que tener en cuenta a la hora de analizar estos datos que en la actualidad coexisten varios subsistemas de Formación Profesional: la Formación Profesional Específica que es la que se imparten en los Institutos de Enseñanza Secundaria, y por 
tanto dependen del Ministerio de Educación y Ciencia, y la Formación Profesional de ámbito laboral

La diferencia fundamental de la nueva Formación Profesional promovida por el actual sistema educativo, es que la formación Profesional Especifica deja de ser una formación propedéutica para convertirse en Formación Profesional Terminal, es decir deja de ser un "camino hacia" para convertirse en formación dirigida a la inserción laboral

En Melilla un dato a tener muy en cuenta es que el 83 por ciento de los parados melillenses tienen una cualificación académica y profesional de primer ciclo de la ESO, graduado escolar o equivalente, hacia abajo. Que a lo sumo el 83 por ciento de los mas de 7000 parados melillenses tienen graduado escolar, primer ciclo de la ESO o estudios inferiores a estos. Esta baja cualificación académica y profesional les dificulta en exceso el intentar acceder al mercado de trabajo, pues además existe escaso tejido productivo en la ciudad de Melilla que pueda absorber parte de esta mano de obra sin cualificar. Se hace por tanto casi indispensable el seguir invirtiendo en formación profesional para que el capital humano vaya mejorando su cualificación y permitiendo su acceso en el mercado de trabajo, ya sea por cuenta ajena o por cuenta propia.

Con la entrada en vigor del Real Decreto 1224/2009 de reconocimiento de las competencias profesionales adquiridas por experiencia laboral, establece un procedimiento único, aplicable en el ámbito de la Administración educativa y laboral, que permitirá una convocatoria de ámbito nacional para acreditar la experiencia laboral y la formación de profesionales y, sobre todo, con la entrada en vigor del Real Decreto que regula la aparición de los Centros Integrados de Formación Profesional, es importante reseñar que estas normas van a permitir, entre otras, cosas la homologación entre cursos de Formación Profesional de ámbito laboral y módulos correspondientes a los ciclos formativos de grado medio y ciclos formativos de grado superior correspondientes a la formación profesional especifica. Con lo que un desempleado que no tenga formación académica pero si ocupacional podrá en parte homologar parte de estos estudios de cursos ocupacionales por módulos de ciclos formativos.. La unidad de cambio entre la realización de un curso para desempleados-ocupados y un ciclo formativo, es la denominada UNIDAD DE COMPETENCIA. Esto podría convertirse en algo muy positivo en la Ciudad de Melilla pues dada la estructura del paro en la ciudad, la aparición de centros integrados y su regulación permitiría a través de la realización de cursos de formación ocupacionales el que determinadas personas que cumplieran los requisitos previstos en el RD de reconocimiento de competencias obtuviesen además un titulo académico, con lo que se conseguiría cualificar académicamente a parte de la población en paro a partir de la Formación Profesional para el Empleo

En la actualidad la nueva modalidad de Formación Profesional para el empleo, supone la ejecución de cursos de formación en la que van a coexistir dentro de los 
asistentes a los cursos, tanto los desempleados como los trabajadores ocupados, dependiendo que la modalidad sea de oferta o de demanda, va a suponer que el mayor porcentaje de asistentes ( $60 \%$ ) sea desempleados u ocupados, según la modalidad sea de oferta o de demanda. La aparición de este Real Decreto que regula la Formación Profesional para el empleo, supone la aparición en cadena de las Ordenes Ministeriales que desarrollan la aparición de esta norma, siendo en la actualidad una época efervescente por la aparición continua de normativa ante el cambio de la norma fundamental que regula la formación profesional financiada por el Servicio Público de Empleo Estatal.

También es necesario destacar que la nueva Formación Profesional para el Empleo, que se imparte con Fondos del presupuesto de gastos del SPEE, resulta que también son cofinanciados por el Fondo Social Europeo, durante el próximo programa Operativo para el septenio 2007-2013, gracias al Programa Operativo "Adaptabilidad y Empleo" del F.S.E. , el porcentaje de cofinanciación de las Políticas Activas relacionados con la Formación Profesional para el Empleo es de un $80 \%$ para la ciudad autónoma de Melilla. Este elevado porcentaje de cofinanciación es debido a que Melilla se considera una región "PHASING OUT", esto quiere decir que el Producto Interior Bruto per cápita de un melillense es inferior a la media del PIP per cápita de la Unión Europea de los quince primeros países miembros de la Unión Europea, sin embargo el PIP per cápita de un ciudadano melillense es superior a la media del PIP per cápita de un ciudadano de la Unión Europea de los 25 países miembros, es decir, después de la última ampliación.

Esta importante cofinanciación va a conseguir que el número de cursos que se destinen a desempleados y a trabajadores en activo u ocupados durante el septenio 2007-2013 sea bastante elevado. Por ello es fundamental que los grandes esfuerzos que se hacen desde el punto de vista presupuestario en las políticas de formación tengan incidencia en la configuración del mercado de trabajo en la ciudad, sobretodo en lo relativo al aumento de la cualificación profesional de desempleados y ocupados, y también un aumento en la mejora de los índices de inserción profesional de los cursos que se desarrollen en este periodo.

\section{LINEAS DE INVESTIGACIÓN FUTURAS Y COMPLEMENTARIAS AL ACTUAL PROYECTO DE INVESTIGACIÓN.}

Este proyecto de investigación NO pretende ser un instrumento estático, puesto que sino perdería su validez, debe ser un instrumento que permita analizar la validez del actual subsistema de Formación Profesional para el empleo en una Ciudad Autónoma con unas tasas de desempleo de las más altas del país. Además el periodo convulso desde el punto de vista normativo en todo lo relativo a la Formación Profesional hace 
más que necesario posteriores evaluaciones de los nuevos subsistemas de Formación que se están implantando. Por todo ello algunas de las líneas de investigación que tendrían relación con el actual proyecto de investigación serian:

$1^{\circ}$.- Evaluación de la Formación del Servicio Publico de Empleo Estatal después de la implantación de la nueva normativa derivada de la entrada en vigor de la Formación Profesional para el Empleo

$2^{\circ}$.- Comparativa de los parámetros de calidad y de inserción profesional de los cursos formación en la ciudad de Melilla antes y después de la entrada en vigor de la Formación Profesional para el empleo

$3^{\circ}$.- Evaluación de la calidad de la Formación Profesional Específica impartida por el MEC en los diferentes Institutos de Enseñanza Secundaria de la ciudad autónoma de Melilla

$4^{\mathrm{o}}$.- Estudios de correlación entre mejora de la cualificación del mercado de trabajo, inserción profesional y nivel de gasto público en formación profesional cofinanciada por el Fondo Social Europeo.

5.- Determinación de ratios para evaluar la eficacia y eficiencia del gasto publico en formación profesional

6.- Estudio de necesidades formativas y Mapa de la Formación Profesional en la Ciudad Autónoma de Melilla

$7^{\circ}$.- Estudio comparativo de la inserción laboral en la Formación Profesional para el empleo con la anterior normativa laboral

\section{REFERENCIAS BIBLIOGRÁFICAS}

Alex, L. (1991): "Descripción y registro de las cualificaciones. El concepto de cualificación”. En Formación Profesional, 2, pp. 23-27.

Bunk, G.P. (1994): "La transmisión de las competencias en la formación y perfeccionamiento profesionales de la RFA". En Revista Europea de Formación Profesional, 1, pp. $8-14$.

Echeverría, B. (2000): "Macrotendencias" de la Formación Profesional en la Unión Europea. Barcelona.

Mertens, D.M. (1998): Research Methods in Education and Psychology. Thousands Oaks (USA), Sage.

Ministerio de Trabajo y Asuntos Sociales (2007): Base de datos trabajadores afiliados en alta laboral por actividad económica (CNAE 93) y base de datos del número de empresas en alta por actividad económica (CNAE 93). Melilla. 
Real Decreto 1224/2009 de reconocimiento de las competencias profesionales adquiridas por experiencia laboral,

Real Decreto 676/1993, de 7 de mayo, por el que se establecen directrices generales sobre los títulos y las correspondientes enseñanzas mínimas de formación profesional. (BOE 122/93 de 22 de mayo).

Real Decreto 797/1995 de 19 de mayo, por el que se establecen directrices sobre los Certificados de Profesionalidad y los correspondientes contenidos mínimos de formación profesional. (BOE nº 138 de 10 de junio de 1995).

Real Decreto 631/1993, de 3 de mayo, por el que se regula el Plan Nacional de Formación e Inserción Profesional. (BOE 4/05/93).

Zabala y Arnau 2007. Como aprender y enseñar competencias. 11 ideas clave 